\title{
Genomic analysis of bacteriophage Xoo-sp13 infecting Xanthomonas oryzae pv. oryzae
}

\author{
Amina Nazir ${ }^{1,2}\left(\right.$ Zhaoxia Dong $^{3} \cdot$ Jin Liu $^{3} \cdot$ Rana Adnan Tahir ${ }^{1} \cdot$ Madiha Rasheed $^{4} \cdot$ Hong Qing $^{1} \cdot$ Donghai Peng $^{3} \cdot$ \\ Yigang Tong ${ }^{2}$
}

Received: 24 September 2020 / Accepted: 21 December 2020 / Published online: 14 February 2021

(C) The Author(s), under exclusive licence to Springer-Verlag GmbH, AT part of Springer Nature 2021

\begin{abstract}
Xanthomonas oryzae pv. oryzae is a bacterial pathogen that gives rise to diseases in rice all over the world. A bacteriophage infecting this bacterium was isolated from rice fields in China. Here, we report the complete genome sequence of this phage, which has a linear dsDNA genome of 309,023 bp and a $\mathrm{G}+\mathrm{C}$ content of $42.43 \%$. It contains 401 open reading frames and encodes 28 tRNAs. It belongs to the family Myoviridae and has a broad host range, making it a possible candidate for phage therapy.
\end{abstract}

Xanthomonas oryzae pv. oryzae is considered the most important agent of bacterial blight of rice [1], a disease that can cause major crop destruction and threatens global food security [10]. Bacteriophages are ubiquitous in the environment, and some have the potential to control bacterial diseases [2]. Therefore, various strategies have been developed

Handling Editor: T. K. Frey.

Amina Nazir, Zhaoxia Dong and Jin Liu contributed equally.

Hong Qing

donghaipeng@mail.hzau.edu.cn

$\triangle$ Donghai Peng

hqing@bit.edu.cn

Yigang Tong

tong.yigang@gmail.com

1 Key Laboratory of Molecular Medicine and Biotherapy in the Ministry of Industry and Information Technology, Department of Biology, School of Life Sciences, Beijing Institute of Technology, Beijing, People's Republic of China

2 State Key Laboratory of Pathogen and Biosecurity, Beijing Institute of Microbiology and Epidemiology, Beijing 100071, People's Republic of China

3 State Key Laboratory of Agricultural Microbiology, College of Life Science and Technology, Huazhong Agricultural University, Wuhan 430070, Hubei, People's Republic of China

$4 \quad$ Beijing Key Laboratory for Separation and Analysis in Biomedicine and Pharmaceuticals, School of Life Sciences, Beijing Institute of Technology, Beijing, People's Republic of China to control pathogens by utilizing either a single phage or a cocktail of phages [3]. To gain further insights into the genetic diversity of Xanthomonas phages, we previously isolated a novel phage, Xoo-sp13, a polyvalent phage with a wide spectrum of activity and potential as a biocontrol agent against $X$. oryzae. This phage may be useful for curing bacterial diseases and other biotechnological applications.

Phage Xoo-sp13 was isolated from a soil sample in Shandong, East China, using the PXO99A strain, which was derived from Philippine race 6 strain PXO99, as a host. Methods for isolation, purification, and the host range determination of phage Xoo-sp13 were described previously [4]. The phage was visualized by transmission electron microscopy (TEM) at $200 \mathrm{kV}$, and images were produced at the Wuhan Institute of Virology, China. Phage genomic DNA was extracted using $\mathrm{ZnCl}_{2}$ precipitation [5]. A phage sequencing library was prepared using a NEBNext Ultra II kit v3 (New England Biolabs). Whole-genome sequencing of the phage was performed by Berry Genomics Biotechnology Co., Ltd (Beijing, China) using Illumina HiSeq 2500 paired-end sequencing technology. The complete genome sequence of Xoo-sp13 was annotated using Rapid Annotation using Subsystem Technology (RAST; http://rast.nmpdr .org) [6]. All predicted ORFs were checked manually against the National Center for Biotechnology Information (NCBI) database (https://www.ncbi.nlm.nih.gov/) using PSI-BLAST $(E$-value $=0.0001)$ [7]. tRNAscan-SE (http://lowelab.ucsc. edu/tRNAscan-SE/index.html) was used for the prediction of genes encoding tRNAs [8]. 


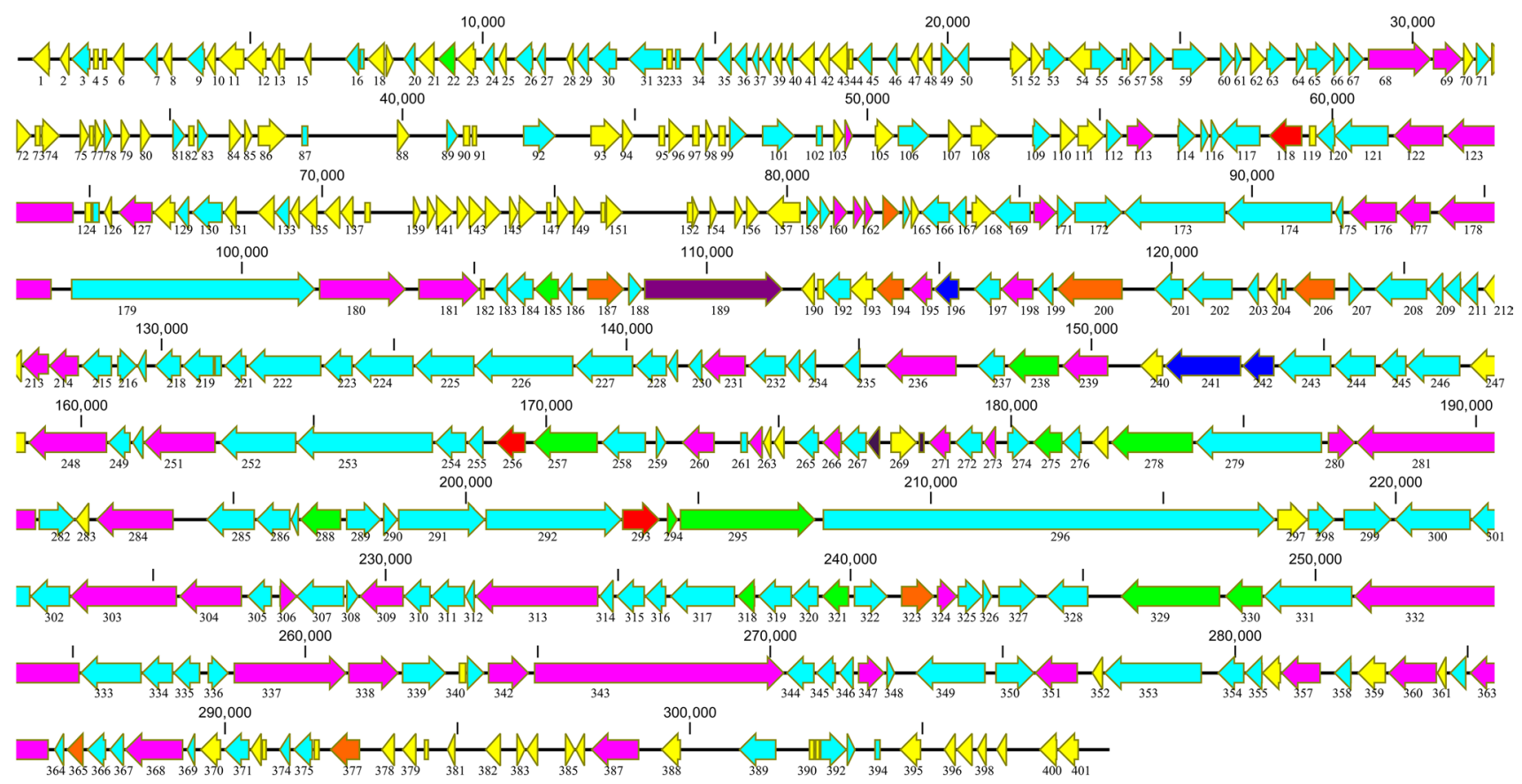

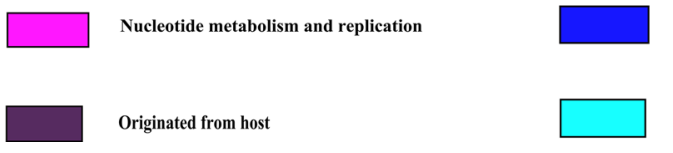

Fig. 1. Schematic representation of the dsDNA genome of phage Xoo-sp13. Putative ORFs are presented as arrows, with predicted functions where available. Proposed modules are based on predicted functions. Turquoise, hypothetical protein; yellow, unknown; pink,

TEM results showed that Xoo-sp13 has an isometric head $(60.56 \pm 3 \mathrm{~nm})$ and a contractile tail $(121.56 \pm 2$ $\mathrm{nm})$. Its genome sequence and morphology suggest that it belongs to the family Myoviridae, based on the current International Committee on Taxonomy of Viruses classification system (Fig. S1). A comparison of the genome sequence of Xoo-sp13 with other sequences in the GenBank Database using BLASTn showed it to be $75 \%$ identical to Xanthomonas phage XacN1 (accession no. AP018399.1). Phage XacN1 was isolated from a soil sample and infects Xanthomonas citri, a causative agent of Asian citrus canker. Xoo-sp13 had a linear dsDNA genome of $309,023 \mathrm{bp}$ and a $\mathrm{G}+\mathrm{C}$ content of $42.43 \%$. Interestingly, Xoo-sp13 possesses unique characteristics due to the presence of a long terminal repeat of about $50 \mathrm{~kb}$, which was detected as described previously [9]. A total of 401 ORFs and 28 tRNAs were found in the genome of Xoo-sp13 (Table S1). These include 135 genes that are unrelated to any genes from the prokaryotic or viral database and 188 that have been annotated as hypothetical proteins. Only 79 ORFs were identified as encoding proteins with known functions (Table S2), thus highlighting the novelty of this nucleotide metabolism and replication; green, Morphogenesis; red, lysis; blue, DNA packaging. The map was drawn with CLC Genomics main Workbench version 3.6.1 (CLC bio, Aarhus, Denmark)

phage (Fig. 1). Phage Xoo-sp13 was tested against sixteen different strains of $X$. oryzae for host range analysis and found to infect 9 of them (Table S3), indicating a broad host range.

In conclusion, Xoo-sp13 is a bacteriophage with a large DNA genome and low sequence similarity to other known phages. It represents a new addition to the list of $X$. oryzae phages. Interestingly, most of the open reading frames in its genome are not functionally annotated. Determining the functions of these genes will be an exciting subject of study for understanding the biology of this novel phage.

\section{Nucleotide sequence accession number}

The complete genome sequence of phage Xoo-sp13 with annotations was submitted to the GenBank database under the accession number MN047793.

Supplementary Information The online version contains supplementary material available at https://doi.org/10.1007/s00705-021-04985-4. 
Funding This research was supported by a grant from the National Key Research and Development Program of China (2018YFA0903000), the National Natural Science Foundation of China (81672001), and the China MoST Emergency Project on COVID-19 (2020YFC0840800).

\section{Compliance with ethical standards}

Conflict of interest The authors declare that they have no conflict of interest.

Ethical approval This article does not contain any studies with human participants or animals performed by any of the authors.

\section{References}

1. Liu W, Liu J, Triplett L, Leach JE, Wang G-L (2014) Novel insights into rice innate immunity against bacterial and fungal pathogens. Annu Rev Phytopathol 52:213-241

2. Xi H, Dai J, Tong Y, Cheng M, Zhao F, Fan H, Li X, Cai R, Ji Y, Sun C (2019) The characteristics and genome analysis of vB_AviM_AVP, the first phage infecting Aerococcus viridans. Viruses 11(2):104

3. Kim MS, Hong SS, Park K, Myung H (2013) Genomic analysis of bacteriophage PBECO4 infecting Escherichia coli O157: H7. Adv Virol 158(11):2399-2403

4. Dong Z, Xing S, Liu J, Tang X, Ruan L, Sun M, Tong Y, Peng D (2018) Isolation and characterization of a novel phage
Xoo-sp2 that infects Xanthomonas oryzae pv. oryzae. J Gen Virol 99(10): 1453-1462

5. MrA S (1991) An improved method for the small scale preparation of bacteriophage DNA based on phage precipitation by zinc chloride. Nucleic Acids Res 19(19):5442

6. Aziz RK, Bartels D, Best AA, DeJongh M, Disz T, Edwards RA, Formsma K, Gerdes S, Glass EM, Kubal M (2008) The RAST Server: rapid annotations using subsystems technology. BMC Genom 9(1):75

7. Stephen FA (1997) Gapped BLAST and PSI-BLAST: a new generation of protein database search programs. Nucleic Acids Res 25:3389-3402

8. Schattner P, Brooks AN, Lowe TM (2005) The tRNAscan-SE, snoscan and snoGPS web servers for the detection of tRNAs and snoRNAs. Nucleic Acids Res 33(suppl_2):W686-W689

9. Zhang X, Wang Y, Tong Y (2018) Analyzing genome termini of bacteriophage through high-throughput sequencing. In: Clokie Martha RJ et al (eds) Bacteriophages: Methods and Protocols, vol 3. Springer, pp 139-163

10. Nazir A, Dong Z, Liu J, Zhang X, Tahir RA, Ashraf N, Qing H, Peng D, Tong Y (2020) Sequence analysis of a jumbo bacteriophage, Xoo-sp14, that infects Xanthomonas oryzae pv. oryzae. Microbiol Resour Announc 9:e01072-e1120

Publisher's Note Springer Nature remains neutral with regard to jurisdictional claims in published maps and institutional affiliations. 